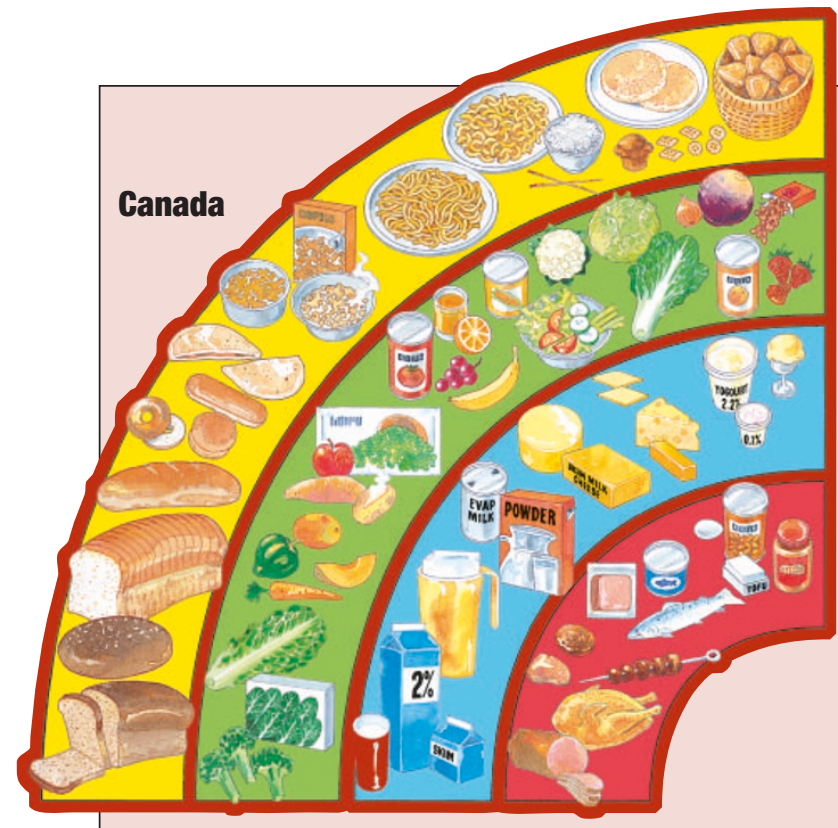

\title{
Around the world in three square meals
}

In China, it's a pagoda; in Canada, a rainbow.

But despite the diversity of design to be found in pictorial food guides from around the world, the core advice remains the same: eat your peas and porridge, limit your bacon and eggs.

In Canada's rainbow, for instance, breads and cereals occupy the outermost - and therefore longest - curve of the arc. This band is coloured golden yellow to represent grain. Vegetables and fruit are next in green, followed by dairy in blue and meat in the diminutive, innermost red band.

Of course, in real rainbows, even Canadian ones, red comes before yellow. Putting the colours in that order comes a bit closer to what real Canadians eat. According to data from the United Nations Food and Agriculture Organization, Canadians, like other North Americans, get more than twice as many calories from meat and fish as from fruit and vegetables.

That ratio is fairly common in parts of the world where meat is readily available. The Chinese, avid consumers of pork, have a similar proportion in their diets. This is despite the advice of the Food Guide Pagoda, which has grain in its foundation level, and fruit and veg just above it.

Nearly every official food guide emphasizes grains and cereals as the foundation of a healthy diet, and that's one recommendation the world as a whole has no trouble living up to. In the United States and Europe, grains and cereals make up about a quarter of the average diet. In Asia, where rice is a staple, they are anywhere from $50 \%$ to $60 \%$ of daily calories. Diets in most other regions fall somewhere in the middle.

Although the basic recommendations are the same, each pyramid, rainbow or circle tends to reflect the nation's unique food culture. The Mexican food circle has an entire wedge devoted to beans. The Chinese pagoda's food depictions include a bowl of rice and a head of bok choy, and the German food circle features photographs of hearty whole-grain breads.

But no food guide seems to take adequate account of the irrepressible human sweet tooth. Sweets are listed along with fats as only for occasional consumption in most guides. And several make no mention of sweets at all, including those from China, Sweden, Germany and Portugal (see J. Painter, J.-H. Rah and Y.-K. Lee J. Am. Diet. Assoc. 102, 483-489; 2002). Even so, North Africans get $9 \%$ of their calories from sugar, Europeans $11 \%$, and Americans a cloying $18 \%$.

Jonathan Knight 